\title{
Self-consistent determination of the perpendicular strain profile of implanted Si by analysis of X-ray rocking curves
}

\author{
C. J. Tsai, A. Dommann, ${ }^{\text {a) }}$ M. A. Nicolet, and T. Vreeland, Jr. \\ California Institute of Technology, Pasadena, California 91125
}

(Received 21 September 1990; accepted for publication 31 October 1990)

\begin{abstract}
Results of a determination of strain perpendicular to the surface and of the damage in (100)
Si single crystals irradiated by $250-\mathrm{keV} \mathrm{Ar}^{+}$ions at $77 \mathrm{~K}$ are presented. Double-crystal $\mathrm{x}$-ray diffraction and dynamical $\mathrm{x}$-ray diffraction theory are used. Trial strain and damage distributions were guided by transmission electron microscope observations and Monte Carlo simulation of ion energy deposition. The perpendicular strain and damage profiles, determined after sequentially removing thin layers of $\mathrm{Ar}^{+}$-implanted $\mathrm{Si}$, were shown to be self-consistent, proving the uniqueness of the deconvolution. Agreement between calculated and experimental rocking curves is obtained with strain and damage distributions which closely follow the shape of the TRIM simulations from the maximum damage to the end of the ion range but fall off more rapidly than the simulation curve near the surface. Comparison of the TRIM simulation and the strain profile of $\mathrm{Ar}^{+}$-implanted Si reveals the importance of annealing during and after implantation and the role of complex defects in the final residual strain distribution.
\end{abstract}

\section{INTRODUCTION}

The $\mathrm{x}$-ray rocking curve method is a useful tool to determine the strain in a crystal. The method consist of matching rocking curves that are calculated from assumed depth profiles of strain and damage with the measured rocking curves obtained from crystal samples. The problem is that a good match is a necessary, but not sufficient condition to identify the actual profiles.

The strain sensitivity of the $x$-ray rocking curve method is limited by instrument broadening of the diffraction peaks and by broadening due to crystal defects. In the case where the strained layers are so thin that the kinematical theory of $x$-ray diffraction ${ }^{1}$ is applicable, the difference between the $\mathrm{x}$-ray rocking curves of two strained layers $\mathrm{A}$ and $B$ observed in the stacking order $\mathbf{A B}$ or $\mathbf{B A}$ is difficult to detect. In this case, the rocking curve can be considered as a simple superposition of the diffraction peaks of the two layers and the substrate peak. The rocking curves from the $\mathrm{AB}$ and $\mathrm{BA}$ sequences are indistinguishable when the $\mathrm{x}$-ray absorption is small. In the case when the strain layers are thick enough so that the dynamical theory of $\mathrm{x}$-ray diffraction $^{2,3}$ should be applied, crystal defects and instrumental broadening may still lead to the same problem. Figure 1 shows the two rocking curves for samples with the two layer sequences $\mathrm{AB}$ and $\mathrm{BA}$ calculated with the dynamical theory for $\mathrm{Si}(400)$ diffraction using an $\mathrm{FeK}_{\alpha 1}$ (wavelength $0.1936 \mathrm{~nm}$ ) incident beam. The calculated rocking curves were convoluted with a Lorentzian function of 6 arcsec full width half maximum (to account for instrument broadening). In Fig. 1(c), we can see only a small difference in the two curves. As the broadening increases, the difference becomes smaller and smaller. Complicated strain profiles such as those of ion-implanted crystals must therefore be carefully analyzed to determine the actual strain profile. A damage profile is required to match the $\mathrm{x}$-ray intensities from strained layers of implanted crystals. ${ }^{4}$

Speriosu and co-workers ${ }^{5}$ have employed a sequential etching process to assure the correctness of the strain and damage distributions in ion-implanted magnetic bubble materials using $x$-ray rocking curves and the kinematical theory of $x$-ray diffraction. We report on a combination of transmission electron microscopy (TEM) study, computer simulation, and sequential etching technique which permits a unique deconvolution of strain and damage profiles from the $\mathrm{x}$-ray rocking curves of ion-implanted samples.

\section{EXPERIMENT}

A (100) silicon wafer of $0.4 \mathrm{~mm}$ thickness was irradiated on its poiished side at $77 \mathrm{~K}$ with $250-\mathrm{keV} \mathrm{Ar}^{+}$ions and a beam flux of $<0.1 \mu \mathrm{A} / \mathrm{cm}^{2}$ in a vacuum of $<10^{-6}$ Torr to a dose of $4 \times 10^{13}$ ions $/ \mathrm{cm}^{2}$. The sample was tilted $7^{\circ}$ to avoid the channelling effect. The implanted sample was stored at room temperature for one month to minimize further room- temperature annealing during $\mathrm{x}$-ray analysis. $\mathrm{X}$-ray rocking curves using (400) and (333) reflections were then taken. The sample was then etched in diluted CP4 ( $5 \mathrm{HNO}_{3}: 3 \mathrm{HF}: 3 \mathrm{CH}_{3} \mathrm{COOH}: 44 \mathrm{H}_{2} \mathrm{O}$ ) for $2 \mathrm{~s}$ in five steps. X-ray rocking curves were taken after each etching step. Other samples with larger doses of $250-\mathrm{keV} \mathrm{Ar}^{+}$, up to $5 \times 10^{16}$ ions $/ \mathrm{cm}^{2}$, were used to find the range of strain and damage. The highest dose was sufficient to amorphize all of the damaged region. The depth of this amorphization, determined using cross-sectional transmission clectron microscopy (XTEM), marked the extent of the strained and damaged region. Contrast in XTEM micrographs of a sample given a dose of $5 \times 10^{13}$ ions $/ \mathrm{cm}^{2}$, re-

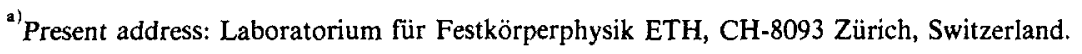



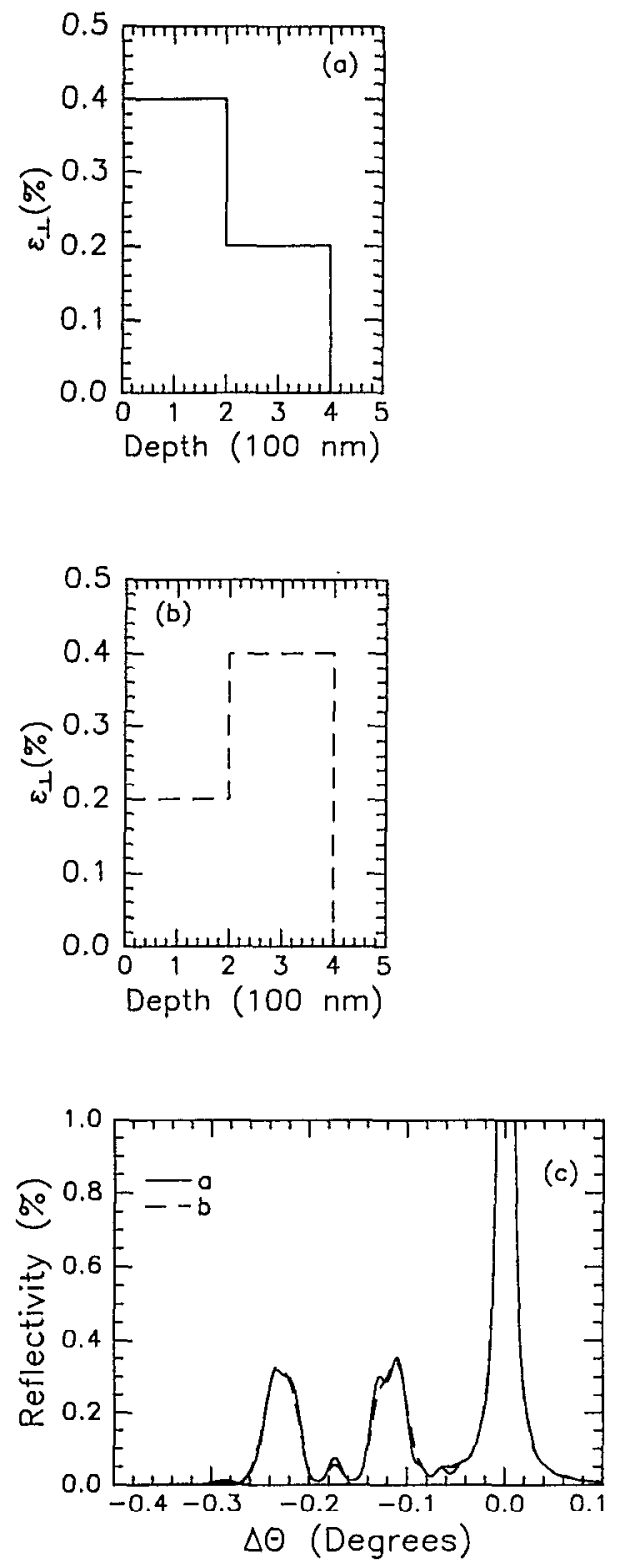

FIG. 1. Calculated $\mathrm{FeK}_{\alpha 1}$ (400) rocking curves for (100) Si with the strain distributions shown in (a) and (b).

sulting from point defect clusters, marked the region of highest damage (Fig. 2).

\section{RESULTS AND DISCUSSION}

A direct measurement of the removed layer thickness by a stencil technique was unreliable due to the lack of sharp steps. Removal of layers of oxide, produced by anodic anodization and measured by RBS, did not give the desired sensitivity. Although the influence of strain on the etching rate is unknown, we assume that the thickness of the removed layer in each etching step is the same. The thickness was derived by reference to Fig. 2 and the $x$-ray rocking curves as follows. The $\mathrm{x}$-ray rocking curves taken after each etching step are shown in Fig. 3(a). Note that the first etching reduces the reflectivity at small angular shifts from the substrate peak (from 0 to -0.05 degrees),

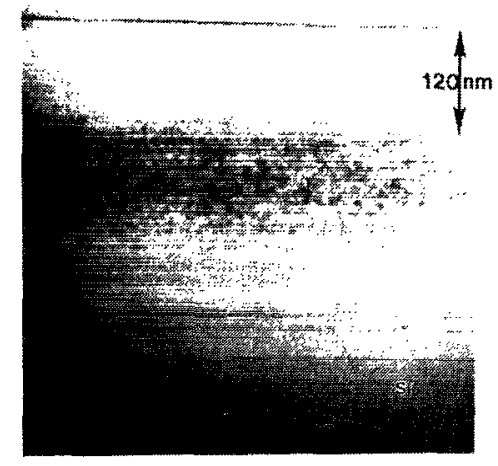

FIG. 2. XTEM micrograph of a (100) Si wafer implanted with $5 \times 10^{13}$ $\mathrm{Ar}^{+} / \mathrm{cm}^{2}$ at $77^{\circ} \mathrm{K}$, revealing the region of highest damage between 120 $\mathrm{nm}$ and $240 \mathrm{~nm}$.

leaving the remainder of the curve unaffected. This result indicates that there is a region of small strain near the surface leaving the large strain region unaltered. The region of highest damage is known from Fig. 2 to be located
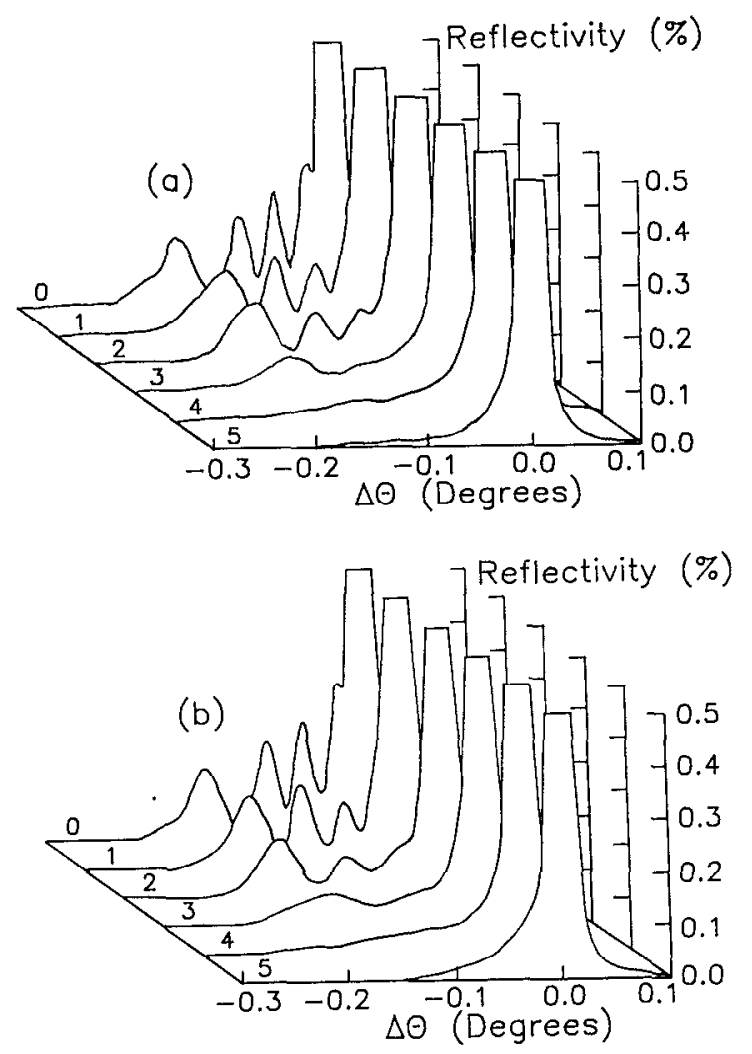

FIG. 3. Experimental (a) and calculated (b) rocking curves for a (100) Si wafer implanted to a dose of $4 \times 10^{13} \mathrm{Ar}^{+} / \mathrm{cm}^{2}$ with $250 \mathrm{KeV} \mathrm{Ar}+$ ions and analyzed with $\mathrm{FeK}_{\alpha 1}(400)$ x-ray diffraction. The numbers $(0-5)$ correspond to the etching steps. 


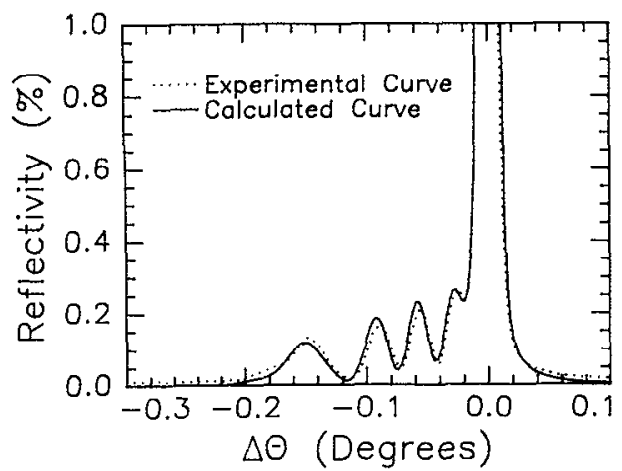

FIG. 4. The calculated (dash line) and the experimental (solid line) rocking curve before etching.

at 120 to $240 \mathrm{~nm}$ below the surface. The region of maximum strain begins to be removed in the third etching step and is almost completely removed at the fifth step, as seen in Fig. 3(a) by the decrease in intensity of the maximum strain peak at -0.15 degrees. We therefore estimate that the maximum strain was located at $180 \mathrm{~nm}$, in the middle of highest damage region. This indicates the depth removed per etching step was $\approx 50 \mathrm{~nm} \pm 10 \%$. The maximum range of strain and damage is about $420 \mathrm{~nm}$, which is the depth of the amorphized region observed in XTEM micrographs of the sample with a $5 \times 10^{16} \mathrm{Ar}^{+} / \mathrm{cm}^{2}$ dose $\left(1.25 \times 10^{3}\right.$ higher than the dose given the sample analyzed with $\mathrm{x}$-rays). The thickness of the strained layer used for fitting the last rocking curve was then $170 \mathrm{~nm}(420-5 \times 50$ $\mathrm{nm}$ ). Strain and damage profiles in this layer, following the shape of the TRIM profile, were then found which gave a calculated rocking curve which fit the last rocking curve. For each additional 50-nm layer added to the surface of this sample, a new simulation was made with the strain and damage in only this layer being adjusted to give a best match to the corresponding experimental rocking curve. Figure 3(b) shows the resulting x-ray rocking curves obtained in this fashion for each etching step. Figure 4 shows the overlapping of the simulated rocking curve with the experimental rocking curve taken before etching. Final self-consistent strain and damage profiles for the entire implanted sample derived in this manner are shown in Fig. 5. Analysis of the (333) rocking curve of the as-implanted sample found no detectable parallel strain.

Paine and co-workers ${ }^{6}$ studied the strain produced by low-dose room-temperature implantations in (100) GaAs. The x-ray strain perpendicular to the crystal surface, $\epsilon^{\perp}$, was found to follow the relationship

$$
\epsilon^{\perp}(x)=K \phi F_{D}(x),
$$

where $F_{D}(x)$ is the average energy per ion per unit depth deposited by nuclear collisions at depth $x, \phi$ is the irradiation dose, and $K$ is a constant. To compare $F_{D}(\mathrm{x})$ with the self-consistent profiles derived here, Fig. 5 also shows $F_{D}(x)$, simulated by TRIM $^{7}$. The strain profile follows closely the curve of $F_{D}$ beyond the maximum strain with a

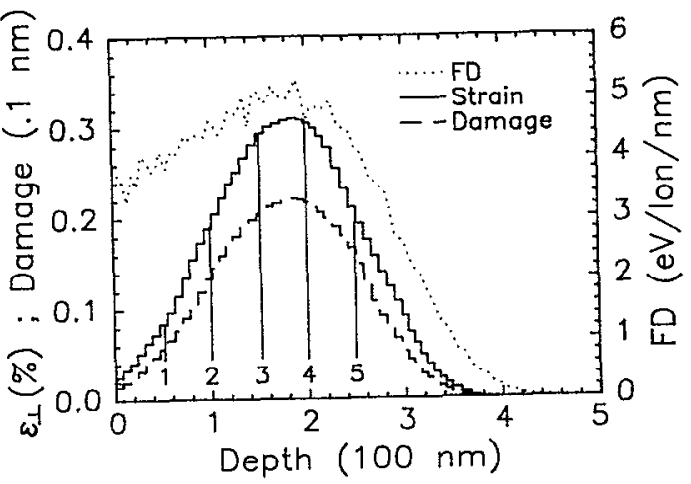

FIG. 5. The self-consistent strain (solid line) and damage (dot-dash line) profiles which give the calculated rocking curve labeled 0 in Fig. 3, and $F_{D}(x)$ (dotted line). The depth of each etching is indicated by a vertical line.

small shift in depth which is within the uncertainty of the depth removed by etching. The lower values of strain and damage toward the surface, as compared to $F_{D}$, suggests that the surface acts as a sink for defects.

Simple vacancies in $\mathrm{Si}$ are mobile at temperatures as low as $70 \mathrm{~K}$ and interstitials are mobile at even lower temperatures. ${ }^{8}$ Defect complexes will have lower mobilities at room temperature, and these defects rather than the simple defects must be responsible for the majority of the residual strain and damage observed. Room- temperature annealing of some complex defects in ion implanted $\mathrm{Si}$ is indicated, so that annealing both during $77 \mathrm{~K}$ implantation and after implantation play an important role in affecting the final strain and damage profiles in $\mathrm{Si}$. The configuration of the complex defects depends on the impurity concentration of the silicon, dopant species and concentration, the implantation temperature, ion energy, ion species, implantation dose, and annealing treatments. Further investigations at an atomic scale are needed to resolve the structure of the defects which produce the strain. Annealing during implantation and post-implantation room-temperature annealing in Si cause the strain to deviate from that given by Eq. (1) in the region between the surface and the depth of maximum damage.

In conclusion, unique strain and damage profiles in ion implanted $\mathrm{Si}$ were determined by $\mathrm{x}$-ray rocking curves combined with etching, XTEM observations, and TRIM simulation. Significant room-temperature annealing in the near-surface regions of Si implanted at liquid-nitrogen temperature are observed. In the simulation of $\mathrm{x}$-ray rocking curves, attention must be given to the fact that profiles of strain and damage which give a calculated rocking curve that matches an experimental rocking curve are not, in general, unique. The correct strain and damage profiles may be found by assuming they follow the shape of $F_{D}$ vs depth beyond its maximum value, with a monotonic decrease in the strain and damage toward the surface to obtain a good fit to the experimental $x$-ray rocking curve.

\section{ACKNOWLEDGMENTS}

This work was supported by the Semiconductor Research Corporation under Contract No. 87-SJ-100. We 
also acknowledge the support of the Swiss National Science Foundation that provided a fellowship to A. Dommann.

'V. S. Speriosu, J. Appl. Phys. 52, 6094 (1981).

${ }^{2}$ W. H. Zachariasen, Theory of $X$-Ray Diffraction in Crystal (Wiley, New York, 1945).

${ }^{3}$ C. R. Wie, T. A. Tombrello, and T. Vreeland, Jr., J. Appl. Phys. 59, 3743 (1986).
${ }^{4}$ V. S. Speriosu and T. Vreeland, Jr., J. Appl. Phys. 61, 1335 (1984).

${ }^{5}$ V. S. Speriosu, H. L. Glass, and T. Kobayashi, Appl. Phys. Lett. 34, 539 (1979).

${ }^{6}$ B. M. Paine, N. N. Hurvitz, and V. S. Sperious, J. Appl. Phys. 61, 1335 (1987).

${ }^{7}$ J. P. Biersack and L. G. Haggmark, J. Nucl. Instrum. Methods 174, 257 (1980).

${ }^{8} \mathrm{~J}$. Bourgoin and M. Lannoo, Point Defects in Semiconductors II (Springer, Berlin, 1983). 